\title{
On small bases for which 1 has countably many expansions
}

Article

Accepted Version

Zou, Y., Wang, L., Lu, J. and Baker, S. (2016) On small bases for which 1 has countably many expansions. Mathematika, 62 (2). pp. 362-377. ISSN 0025-5793 doi:

https://doi.org/10.1112/S002557931500025X Available at https://centaur.reading.ac.uk/46869/

It is advisable to refer to the publisher's version if you intend to cite from the work. See Guidance on citing.

To link to this article DOI: http://dx.doi.org/10.1112/S002557931500025X

Publisher: London Mathematical Society

All outputs in CentAUR are protected by Intellectual Property Rights law, including copyright law. Copyright and IPR is retained by the creators or other copyright holders. Terms and conditions for use of this material are defined in the End User Agreement.

\section{www.reading.ac.uk/centaur}

\section{CentAUR}

Central Archive at the University of Reading

Reading's research outputs online 


\title{
On small bases for which 1 has countably many expansions
}

\author{
Yuru Zou ${ }^{a},{\text { Lijin } \mathrm{Wang}^{a}, \text { Jian }^{a *}{ }^{a *} \text { Simon Baker }}^{b}$ \\ ${ }^{a}$ College of Mathematics and Computational Science, Shenzhen University, Shenzhen 518060, China \\ ${ }^{b}$ School of Mathematics, The University of Manchester, Oxford Road, Manchester M13 9PL, United Kingdom
}

\begin{abstract}
Let $q \in(1,2)$. A $q$-expansion of a number $x$ in $\left[0, \frac{1}{q-1}\right]$ is a sequence $\left(\delta_{i}\right)_{i=1}^{\infty} \in\{0,1\}^{\mathbb{N}}$ satisfying

$$
x=\sum_{i=1}^{\infty} \frac{\delta_{i}}{q^{i}} .
$$

Let $\mathcal{B}_{\aleph_{0}}$ denote the set of $q$ for which there exists $x$ with a countable number of $q$-expansions, and let $\mathcal{B}_{1, \aleph_{0}}$ denote the set of $q$ for which 1 has a countable number of $q$-expansions. In [21] it was shown that $\min \mathcal{B}_{\aleph_{0}}=\min \mathcal{B}_{1, \aleph_{0}}=\frac{1+\sqrt{5}}{2}$, and in [1] it was shown that $\mathcal{B}_{\aleph_{0}} \cap\left(\frac{1+\sqrt{5}}{2}, q_{1}\right]=\left\{q_{1}\right\}$, where $q_{1}(\approx 1.64541)$ is the positive root of $x^{6}-x^{4}-x^{3}-2 x^{2}-x-1=0$. In this paper we show that the second smallest point of $\mathcal{B}_{1, \aleph_{0}}$ is $q_{3}(\approx 1.68042)$, the positive root of $x^{5}-x^{4}-x^{3}-x+1=0$. Enroute to proving this result we show that $\mathcal{B}_{\aleph_{0}} \cap\left(q_{1}, q_{3}\right]=\left\{q_{2}, q_{3}\right\}$, where $q_{2}(\approx 1.65462)$ is the positive root of $x^{6}-2 x^{4}-x^{3}-1=0$.
\end{abstract}

Key Words: beta-expansion, non-integer base, countable expansions

AMS Subject Classifications: 11A63, 37A45

\section{Introduction}

Let $q \in(1,2)$ and $I_{q}:=\left[0, \frac{1}{q-1}\right]$. For each $x \in I_{q}$ there exists a sequence $\left(\delta_{i}\right)_{i=1}^{\infty} \in\{0,1\}^{\mathbb{N}}$ such that

$$
x=\sum_{i=1}^{\infty} \frac{\delta_{i}}{q^{i}} .
$$

The sequence $\left(\delta_{i}\right)_{i=1}^{\infty}$ is called a $q$-expansion for $x$. Without confusion, we simplify $\left(\delta_{i}\right)_{i=1}^{\infty}$ as $\left(\delta_{i}\right)$. It is straightforward to show that a real number $x$ has a $q$-expansion if and only if $x \in I_{q}$.

We now introduce some notation. The so-called coding map is defined to be $\Pi:\{0,1\}^{\mathbb{N}} \rightarrow I_{q}$ where

$$
\Pi\left(\left(\delta_{i}\right)\right)=\sum_{i=1}^{\infty} \frac{\delta_{i}}{q^{i}} .
$$

\footnotetext{
${ }^{*}$ Corresponding author. E-mail:jianlu1979@163.com
} 
Throughout we let $\left(\varepsilon_{1} \ldots \varepsilon_{n}\right)^{k}$ denote the $k$ fold concatenation of $\left(\varepsilon_{1} \ldots \varepsilon_{n}\right) \in\{0,1\}^{n}$, and similarly let $\left(\varepsilon_{1} \ldots \varepsilon_{n}\right)^{\infty}$ denote the infinite concatenations of $\left(\varepsilon_{1} \ldots \varepsilon_{n}\right)$. Given $x \in I_{q}$, let $\Sigma_{q}(x)$ denote the set of all $q$-expansions of $x$, that is

$$
\Sigma_{q}(x)=\left\{\left(\delta_{i}\right) \in\{0,1\}^{\mathbb{N}}: \Pi\left(\left(\delta_{i}\right)\right)=x\right\} .
$$

The cardinality of the set $\Sigma_{q}(x)$ plays an important role in the investigation of representations of real numbers in non-integer bases. It was shown in [11] that if $q \in\left(1, \frac{1+\sqrt{5}}{2}\right)$ then for each $x \in\left(0, \frac{1}{q-1}\right)$ there are $2^{\aleph_{0}}$ different $q$-expansions. Sidorov showed in $[18,19]$ that if $q \in(1,2)$ then Lebesgue almost every $x \in\left(0, \frac{1}{q-1}\right)$ has $2^{\aleph_{0}}$ different $q$-expansions. Points belonging to $I_{q}$ with a unique $q$-expansion were investigated in $[7,15]$ for $q \in\left(\frac{1+\sqrt{5}}{2}, 2\right)$. Some results concerning $x \in I_{q}$ having a fixed number of $q$-expansions were established in $[1,3,8,9,10,20,22]$.

Let $m \in \mathbb{N} \cup\left\{\aleph_{0}\right\}$ and define

$$
\mathcal{B}_{m}:=\left\{q \in\left[\frac{1+\sqrt{5}}{2}, 2\right): \text { there exits } x \in I_{q} \text { satisfying } \# \Sigma_{q}(x)=m\right\} .
$$

Here and hereafter $\# A$ denotes the cardinality of a set $A$. The following results are known to hold:

- $\min \mathcal{B}_{\aleph_{0}}=\frac{1+\sqrt{5}}{2}[10]$

- $\min \mathcal{B}_{2}=\check{q} \approx 1.71064$ (the positive root of $x^{4}-2 x^{2}-x-1=0$ ) [20].

- $\min \mathcal{B}_{k}=q_{f} \approx 1.75488, k \geq 3$ (the positive root of $x^{3}-2 x^{2}-1=0$ ) [3].

- $\mathcal{B}_{2} \cap\left(\check{q}, q_{f}\right]=\left\{q_{f}\right\}[3]$.

- The smallest element of $\mathcal{B}_{\aleph_{0}}$ strictly greater than $\frac{1+\sqrt{5}}{2}$ is $q_{1}(\approx 1.64541)$ (the positive root of $\left.x^{6}-x^{4}-x^{3}-2 x^{2}-x-1=0\right)[1]$.

Understanding the $q$-expansions of 1 is a classical problem, see $[15,16,17]$ and the references therein. The motivation of this paper is to provide a clearer understanding of what values $\# \Sigma_{q}(x)$ can take. Let

$$
\mathcal{B}_{1, m}:=\left\{q \in\left[\frac{1+\sqrt{5}}{2}, 2\right): \# \Sigma_{q}(1)=m\right\} .
$$

It was shown in [16] that $\min \mathcal{B}_{1,1} \approx 1.78723$ (the Komornik-Loreti constant). In [10] it was proved that $\min \mathcal{B}_{1, \aleph_{0}}=\frac{1+\sqrt{5}}{2}$. For any $n \in(\mathbb{N} \backslash\{1\}) \cup\left\{\aleph_{0}\right\}$, Erdös and Joó $[12,13]$ constructed a continuum of real numbers $q \in\left[q_{0}, 2\right)\left(q_{0}>1.99803\right)$ for which the number 1 has precisely $n q$-expansions.

Motivated by the results listed above, a natural question arises: what is the second smallest point of $\mathcal{B}_{1, \aleph_{0}}$ ? In this paper we will answer this question.

Throughout this paper we let $q_{1}, q_{2}, q_{3}$ be as follows: $q_{1} \approx 1.64541, q_{2} \approx 1.65462$ and $q_{3} \approx 1.68042$, which are the positive roots of $x^{6}-x^{4}-x^{3}-2 x^{2}-x-1=0, x^{6}-2 x^{4}-x^{3}-1=0$ and $x^{5}-x^{4}-x^{3}-x+1=0$, respectively. Our main result is the following.

Theorem 1.1. The smallest element of $\mathcal{B}_{1, \aleph_{0}}$ strictly greater than $\frac{1+\sqrt{5}}{2}$ is $q_{3}$. 
Enroute to proving this result we show the following.

Theorem 1.2. $\mathcal{B}_{\aleph_{0}} \cap\left(\frac{1+\sqrt{5}}{2}, q_{3}\right]=\left\{q_{1}, q_{2}, q_{3}\right\}$.

We remark that $\mathcal{B}_{\aleph_{0}} \cap\left(\frac{1+\sqrt{5}}{2}, q_{1}\right]=\left\{q_{1}\right\}$ is known [1]. The following corollary is an immediate consequence of Theorem 1.1, $\min \mathcal{B}_{2} \approx 1.71064[21], \min \mathcal{B}_{k} \approx 1.75488$ for $k \geq 3[3], \mathcal{B}_{2} \cap\left(\check{q}, q_{f}\right]=\left\{q_{f}\right\}[3]$, and $\min \mathcal{B}_{1,1} \approx 1.78723[16]$.

Corollary 1.3. If $q \in\left(1, q_{3}\right) \backslash\left\{\frac{1+\sqrt{5}}{2}\right\}$. Then 1 has $2^{\aleph_{0}}$ different $q$-expansions.

This paper is arranged as follows. Some definitions and results from [1] will be recalled in Section 2. Some results from this paper will be extended to our setting. In Section 3 we prove Theorem 1.2. The final section is devoted to the proof of Theorem 1.1.

\section{Preliminaries}

In this section we shall recall some definitions and results from [1]. An interpretation of $q$-expansions from the perspective of dynamical systems was given in [1], see also $[4,5,6]$. Let $T_{q, 0}(x)=q x$ if $x \in$ $\left[0, \frac{1}{q^{2}-q}\right]$, and let $T_{q, 1}(x)=q x-1$ if $x \in\left[\frac{1}{q}, \frac{1}{q-1}\right]$. We see that if $x \in L_{q}:=\left[0, \frac{1}{q}\right)$ or $x \in R_{q}:=\left(\frac{1}{q^{2}-q}, \frac{1}{q-1}\right]$ then only one $T_{q, i}$ can be applied. However when $x \in S_{q}:=\left[\frac{1}{q}, \frac{1}{q^{2}-q}\right]$, which is usually referred to as the switch region, we have a choice between $T_{q, 0}$ and $T_{q, 1}$. An element of $\bigcup_{n=0}^{\infty}\left\{T_{q, 0}, T_{q, 1}\right\}^{n}$ is denoted by $\mathbf{a}$, here $\left\{T_{q, 0}, T_{q, 1}\right\}^{0}$ denotes the identity map. Moreover, if $\mathbf{a}=a_{1} \ldots a_{n}$ we shall use $\mathbf{a}(x)$ to denote $a_{n}\left(\cdots\left(a_{1}(x)\right) \cdots\right)$. Given $x \in I_{q}$ we call a finite sequence of transformations $\mathbf{a}=a_{1} \ldots a_{n}$ minimal for $x$ if $\mathbf{a}(x) \in S_{q}$ and $\mathbf{a} \mid i(x) \notin S_{q}$ for all $i<n$. Here $\mathbf{a} \mid i=a_{1} \ldots a_{i}$. We call $\mathbf{a}(x)$ a branching point of $x$ if $\mathbf{a}(x) \in S_{q}$.

Define

$$
\Omega_{q}(x):=\left\{\left(a_{i}\right)_{i=1}^{\infty} \in\left\{T_{q, 0}, T_{q, 1}\right\}^{\mathbb{N}}: a_{n}\left(\cdots\left(a_{1}(x)\right) \cdots\right) \in I_{q} \text { for all } n \in \mathbb{N}\right\}
$$

The set $\Omega_{q}(x)$ is significant because $\# \Sigma_{q}(x)=\# \Omega_{q}(x)$, where our bijection is given by mapping $\left(\delta_{i}\right)$ to $\left(T_{q, \delta_{i}}\right)$, see [2].

Construction of the branching tree The branching tree was constructed in [1] to study $\mathcal{B}_{\aleph_{0}}$. We now provide details of its construction. Suppose $x \in I_{q}$ and $\Omega_{q}(x)$ (or $\left.\Sigma_{q}(x)\right)$ is infinite. There exists a unique minimal $\mathbf{a} \in \bigcup_{n=0}^{\infty}\left\{T_{q, 0}, T_{q, 1}\right\}^{n}$ such that $\mathbf{a}(x) \in S_{q}$. Then there are two possibilities.

Case 1: There exists a unique $i \in\{0,1\}$ such that $\Omega_{q}\left(T_{q, i}(\mathbf{a}(x))\right)$ is finite and $\Omega_{q}\left(T_{q, 1-i}(\mathbf{a}(x))\right)$ is infinite. In this case, we draw a horizontal line of finite length that then bifurcates with an upper and lower branch. The lower branch corresponds to $T_{q, i}(\mathbf{a}(x))$ and stops bifurcating, the upper branch corresponds to $T_{q, 1-i}(\mathbf{a}(x))$ and goes on bifurcating.

Case 2: Both $\Omega_{q}\left(T_{q, 0}(\mathbf{a}(x))\right)$ and $\Omega_{q}\left(T_{q, 1}(\mathbf{a}(x))\right)$ are infinite. In this case, we draw a horizontal line of finite length that then bifurcates with an upper and lower branch. The lower branch corresponds to $T_{q, 0}(\mathbf{a}(x))$, the upper branch corresponds to $T_{q, 1}(\mathbf{a}(x))$. Both of them go on bifurcating. 
If $\Omega_{q}\left(T_{q, i}(\mathbf{a}(x))\right)$ is infinite, as in Case 1 or Case 2, then there exists a unique minimal $\mathbf{a}^{\prime} \in \bigcup_{n=0}^{\infty}\left\{T_{q, 0}, T_{q, 1}\right\}^{n}$ such that point $\mathbf{a}^{\prime}\left(T_{q, i}(\mathbf{a}(x))\right)$ goes to Case 1 or Case 2 again. This procedure continues indefinitely. The infinite tree we construct by repeating this process is known as the infinite branching tree corresponding to $x$. Fig. 1 illustrates the bifurcating procedure.

Figure 1: The flow chart of the bifurcating procedure corresponding to $x \in I_{q}$

A point $x$ for which $\Sigma_{q}(x)$ is infinite is said to be a $q$ null infinite point if for each branching point of $x, \mathbf{a}(x)$ never goes to Case 2. It is easy to check that if $x$ is a $q$ null infinite point then $\# \Sigma_{q}(x)=\aleph_{0}$. The $q$ null infinite points have a critical role in the proofs of the main results of [1].

Lemma $\mathbf{A}\left[1\right.$, Proposition 2.7] $q \in \mathcal{B}_{\aleph_{0}}$ if and only if $I_{q}$ contains a $q$ null infinite point

Given $\min \mathcal{B}_{2}=\check{q} \approx 1.71064, \min \mathcal{B}_{k}=q_{f} \approx 1.75488$ for $k \geq 3$, and $\mathcal{B}_{2} \cap\left(\check{q}, q_{f}\right]=\left\{q_{f}\right\}$ we have that if $q \in\left(\frac{1+\sqrt{5}}{2}, q_{f}\right) \backslash\{\check{q}\}$ and $x$ is a $q$ null infinite point, then for each branching point of $x, \mathbf{a}(x)$, we have $\# \Omega_{q}\left(T_{q, i}(\mathbf{a}(x))\right)=\aleph_{0}$ and $\# \Omega_{q}\left(T_{q, 1-i}(\mathbf{a}(x))\right)=1$. Which implies the following inclusion

$$
\left\{\mathbf{a}(x): \mathbf{a}(x) \in S_{q}, \mathbf{a} \in \bigcup_{n=0}^{\infty}\left\{T_{q, 0}, T_{q, 1}\right\}^{n}\right\} \subseteq \bigcup_{i=0}^{1} T_{q, i}^{-1}\left(U_{q}\right) \cap S_{q} .
$$

Here $U_{q}$ denotes the set of $x \in I_{q}$ having a unique $q$-expansion.

Unfortunately, it is difficult to deal with the set $S_{q}$. However, by some deductions we can restrict ourselves to a smaller set $J_{q}:=\left[\frac{q+q^{2}}{q^{4}-1}, \frac{1+q^{3}}{q^{4}-1}\right] \subseteq S_{q} . J_{q}=S_{q}$ if and only if $q=q_{f}$.

Lemma B [1, Lemma 3.1] Let $q \in\left[\frac{1+\sqrt{5}}{2}, q_{f}\right)$. Suppose $x \in I_{q}$ satisfies $\# \Sigma_{q}(x)>1$, then there exists a finite sequence of transformations $\mathbf{a}$ such that $\mathbf{a}(x) \in J_{q}$. Here $q_{f}(\approx 1.75488)$ is the positive root of $x^{3}-2 x^{2}-1=0$.

Proposition 2.2 is devoted to characterizing the set $\bigcup_{i=0}^{1} T_{q, i}^{-1}\left(U_{q}\right) \cap J_{q}$ when $q \in\left[q_{1}, q_{3}\right]$. To prove this proposition we need Lemma C and Lemma 2.1. 
Lemma C [14, Theorem 2] Let $q \in\left(\frac{1+\sqrt{5}}{2}, q_{f}\right)$. Then

$$
U_{q}=\left\{\Pi\left(0^{k}(10)^{\infty}\right), \Pi\left(1^{k}(10)^{\infty}\right), 0, \frac{1}{q-1}\right\} .
$$

Where $k \geq 0$.

Set

$$
\mathbf{y}_{j}=\Pi\left(01^{j}(10)^{\infty}\right) \text { and } \mathbf{z}_{j}=\Pi\left(10^{j}(01)^{\infty}\right)
$$

for $j \geq 1$. Here and hereafter we let $\left(S_{q} \backslash J_{q}\right)_{L}=\left[\frac{1}{q}, \frac{q+q^{2}}{q^{4}-1}\right)$ and $\left(S_{q} \backslash J_{q}\right)_{R}=\left(\frac{1+q^{3}}{q^{4}-1}, \frac{1}{q^{2}-q}\right]$.

Lemma 2.1. Let $q \in\left[q_{1}, q_{3}\right]$. Then the following hold:

(i) $\mathbf{y}_{j} \in J_{q}$ if and only if $\mathbf{z}_{j} \in J_{q}$, and $\mathbf{y}_{j} \in\left(S_{q} \backslash J_{q}\right)_{R}$ if and only if $\mathbf{z}_{j} \in\left(S_{q} \backslash J_{q}\right)_{L}$.

(ii) $T_{q, 0}^{m}\left(T_{q, 1}\left(\mathbf{y}_{j}\right)\right) \in J_{q}$ if and only if $T_{q, 1}^{m}\left(T_{q, 0}\left(\mathbf{z}_{j}\right)\right) \in J_{q}$, and $T_{q, 0}^{m}\left(T_{q, 1}\left(\mathbf{y}_{j}\right)\right) \in\left(S_{q} \backslash J_{q}\right)_{R}$ if and only if $T_{q, 1}^{m}\left(T_{q, 0}\left(\mathbf{z}_{j}\right)\right) \in\left(S_{q} \backslash J_{q}\right)_{L} ;$

(iii) $T_{q, 0}^{m}\left(T_{q, 1}\left(\mathbf{y}_{j}\right)\right)=\mathbf{y}_{k}$ if and only if $T_{q, 1}^{m}\left(T_{q, 0}\left(\mathbf{z}_{j}\right)\right)=\mathbf{z}_{k}$, and $T_{q, 0}^{m}\left(T_{q, 1}\left(\mathbf{y}_{j}\right)\right)=\mathbf{z}_{k}$ if and only if $T_{q, 1}^{m}\left(T_{q, 0}\left(\mathbf{z}_{j}\right)\right)=\mathbf{y}_{k}$. Here $j \geq 1$ and $k \geq 1$.

Proof. Direct computation shows the following equations hold.

$$
\begin{gathered}
\mathbf{y}_{j}-\frac{1+q^{3}}{q^{4}-1}=-\mathbf{z}_{j}+\frac{q+q^{2}}{q^{4}-1}, \mathbf{y}_{j}-\frac{q+q^{2}}{q^{4}-1}=\frac{1+q^{3}}{q^{4}-1}-\mathbf{z}_{j}, \frac{1}{q^{2}-q}-\mathbf{y}_{j}=\mathbf{z}_{j}-\frac{1}{q}, \\
T_{q, 0}^{m}\left(T_{q, 1}\left(\mathbf{y}_{j}\right)\right)-\frac{1+q^{3}}{q^{4}-1}=-T_{q, 1}^{m}\left(T_{q, 0}\left(\mathbf{z}_{j}\right)\right)+\frac{q+q^{2}}{q^{4}-1}, \\
T_{q, 0}^{m}\left(T_{q, 1}\left(\mathbf{y}_{j}\right)\right)-\frac{q+q^{2}}{q^{4}-1}=\frac{1+q^{3}}{q^{4}-1}-T_{q, 1}^{m}\left(T_{q, 0}\left(\mathbf{z}_{j}\right)\right), \frac{1}{q^{2}-q}-T_{q, 0}^{m}\left(T_{q, 1}\left(\mathbf{y}_{j}\right)\right)=T_{q, 1}^{m}\left(T_{q, 0}\left(\mathbf{z}_{j}\right)\right)-\frac{1}{q} .
\end{gathered}
$$

(i) is implied by equation (3), and (ii) is implied by equations (4) and (5).

Simplifying $T_{q, 0}^{m}\left(T_{q, 1}\left(\mathbf{y}_{j}\right)\right)=\mathbf{y}_{k}$ and $T_{q, 1}^{m}\left(T_{q, 0}\left(\mathbf{z}_{j}\right)\right)=\mathbf{z}_{k}$, we see that they are both equivalent to

$$
\left(1+2 q^{-1}-q-q^{-1-j}-q^{-2-m}-q^{-1-m}+q^{-2-k-m}\right)\left(q^{2}-1\right)^{-1}=0 .
$$

Similarly, simplifying $T_{q, 0}^{m}\left(T_{q, 1}\left(\mathbf{y}_{j}\right)\right)=\mathbf{z}_{k}$ and $T_{q, 1}^{m}\left(T_{q, 0}\left(\mathbf{z}_{j}\right)\right)=\mathbf{y}_{k}$, we see that they are both equivalent to

$$
\left(-1-2 q^{-1}+q+q^{-1-j}-q^{-2-m}+q^{-2-k-m}+q^{-m}\right)\left(q^{2}-1\right)^{-1}=0 .
$$

Thus we obtain (iii).

Proposition 2.2. Let $q \in\left[q_{1}, q_{3}\right]$. Then we have

$$
\begin{aligned}
& \bigcup_{i=0}^{1} T_{q, i}^{-1}\left(U_{q}\right) \cap J_{q}=\left\{\mathbf{y}_{j}, \mathbf{z}_{j}, 1 \leq j \leq 3\right\}=\left\{\Pi\left(01^{j}(10)^{\infty}\right), \Pi\left(10^{j}(01)^{\infty}\right), 1 \leq j \leq 3\right\}, \\
& \bigcup_{i=0}^{1} T_{q, i}^{-1}\left(U_{q}\right) \cap\left(S_{q} \backslash J_{q}\right)_{R}=\left\{\mathbf{y}_{j}, j \geq 4,1 /\left(q^{2}-q\right)\right\}=\left\{\Pi\left(01^{j}(10)^{\infty}\right), j \geq 4,1 /\left(q^{2}-q\right)\right\}, \\
& \bigcup_{i=0}^{1} T_{q, i}^{-1}\left(U_{q}\right) \cap\left(S_{q} \backslash J_{q}\right)_{L}=\left\{\mathbf{z}_{j}, j \geq 4,1 / q\right\}=\left\{\Pi\left(10^{j}(01)^{\infty}\right), j \geq 4,1 / q\right\} .
\end{aligned}
$$


Proof. It follows from Lemma $\mathrm{C}$ that

$$
\bigcup_{i=0}^{1} T_{q, i}^{-1}\left(U_{q}\right)=\left\{\Pi\left(0^{j}(10)^{\infty}\right), \Pi\left(01^{j-1}(10)^{\infty}\right), \Pi\left(10^{j-1}(10)^{\infty}\right), \Pi\left(1^{j}(10)^{\infty}\right), 0, \frac{1}{q^{2}-q}, \frac{1}{q}, \frac{1}{q-1}\right\} .
$$

Here $j \geq 1$. Then by some straightforward computation we have

$$
\bigcup_{i=0}^{1} T_{q, i}^{-1}\left(U_{q}\right) \cap S_{q}=\left\{\mathbf{y}_{j}, \mathbf{z}_{j}, j \geq 1, \frac{1}{q^{2}-q}, \frac{1}{q}\right\} .
$$

Next, we prove that

$$
\mathbf{y}_{j}-\frac{1+q^{3}}{q^{4}-1}\left\{\begin{array}{l}
\leq 0 \text { when } j<4 \\
>0 \text { when } j \geq 4
\end{array}\right.
$$

when $q \in\left[q_{1}, q_{3}\right]$. It suffices to show that

$$
q^{-1}+q+q^{2}-q^{3}-q^{-1-j}-q^{1-j}\left\{\begin{array}{l}
\leq 0 \text { when } j<4 \\
>0 \text { when } j \geq 4 .
\end{array}\right.
$$

By noting that

$$
\mathbf{y}_{j}-\frac{1+q^{3}}{q^{4}-1}=\left(q^{-1}+q+q^{2}-q^{3}-q^{-1-j}-q^{1-j}\right)\left(q^{4}-1\right)^{-1},
$$

we see that (8) is equivalent to

$$
\ln \frac{\left(q^{2}+1\right)}{-q^{4}+q^{3}+q^{2}+1}(\ln q)^{-1}\left\{\begin{array}{l}
\geq j \text { when } j<4 \\
<j \text { when } j \geq 4 .
\end{array}\right.
$$

The inequalities in (9) are true when $q \in\left[q_{1}, q_{3}\right]$, since $\ln \frac{\left(q^{2}+1\right)}{-q^{4}+q^{3}+q^{2}+1}(\ln q)^{-1}$ is strictly increasing on the interval $\left[q_{1}, q_{4}\right]$, and $\ln \frac{\left(q_{4}^{2}+1\right)}{-q_{4}^{4}+q_{4}^{3}+q_{4}^{2}+1}\left(\ln q_{4}\right)^{-1}=4$ and $\ln \frac{\left(q_{1}^{2}+1\right)}{-q_{1}^{4}+q_{1}^{3}+q_{1}^{2}+1}\left(\ln q_{1}\right)^{-1}=3$. Here $q_{4}(\approx 1.69784)$ is the positive root of $x^{7}-x^{5}-x^{4}-2 x^{3}-2 x^{2}-x-1=0$. A direct computation shows that $\mathbf{y}_{j} \in\left[\frac{q+q^{2}}{q^{4}-1}, \frac{1}{q^{2}-q}\right]$ for all $j \geq 1$. This statement combined with (7) shows that $\mathbf{y}_{j} \in J_{q}$ if $1 \leq j \leq 3$ and $\mathbf{y}_{j} \in\left(S_{q} \backslash J_{q}\right)_{R}$ if $j \geq 4$. Using (i) of Lemma 2.1, we also have $\mathbf{z}_{j} \in J_{q}$ if $1 \leq j \leq 3$ and $\mathbf{z}_{j} \in\left(S_{q} \backslash J_{q}\right)_{L}$ if $j \geq 4$. Our proof now follows from (6).

\section{Proof of Theorem 1.2}

In this section we shall give a algorithm to find all elements of the set $\mathcal{B}_{\aleph_{0}} \cap\left[q_{1}, q_{3}\right]$. Recall that $\min \mathcal{B}_{2}=\check{q} \approx 1.71064$.

Theorem 3.1. Let $q \in\left[q_{1}, q_{f}\right) \backslash\{\check{q}\}$ and suppose $\bigcup_{i=0}^{1} T_{q, i}^{-1}\left(U_{q}\right) \cap J_{q}$ is a finite set. Then $q \in \mathcal{B}_{\aleph_{0}}$ if and only if there exists $w \in \bigcup_{i=0}^{1} T_{q, i}^{-1}\left(U_{q}\right) \cap J_{q}$ satisfying the following two properties.

(i) There exist a finite sequence of transformations $\mathbf{b} \in \bigcup_{n=0}^{\infty}\left\{T_{q, 0}, T_{q, 1}\right\}^{n}$ such that

$$
\mathbf{b}(w)=w .
$$


(ii) Let $\mathbf{b}$ be as above. Define

$$
B(w):=\left\{\mathbf{b}|i: \mathbf{b}| i(w) \in S_{q}, 1 \leq i \leq|\mathbf{b}|\right\}
$$

Then $B(w) \subset \bigcup_{i=0}^{1} T_{q, i}^{-1}\left(U_{q}\right) \cap S_{q}$. Here $|\mathbf{b}|$ denotes the length of $\mathbf{b}$.

Proof. We begin with the rightwards implication. Suppose $q \in \mathcal{B}_{\aleph_{0}}$. By Lemma A, there exists $x \in$ $\left(0, \frac{1}{q-1}\right)$ such that $x$ is a $q$ null infinite point. Furthermore, we may assume $x \in \bigcup_{i=0}^{1} T_{q, i}^{-1}\left(U_{q}\right) \cap J_{q}$ by Lemma B and equation (2). Repeatedly applying Lemma B and equation (2), there exist $\mathbf{a}^{1}, \ldots, \mathbf{a}^{m} \in$ $\bigcup_{n=0}^{\infty}\left\{T_{q, 0}, T_{q 1}\right\}^{n}$ satisfying

$$
\mathbf{a}^{i}\left(\mathbf{a}^{i-1}\left(\ldots\left(\mathbf{a}^{1}(x)\right) \ldots\right)\right) \in \bigcup_{i=0}^{1} T_{q, i}^{-1}\left(U_{q}\right) \cap J_{q}
$$

for each $1 \leq i \leq m$. Note that the set $\bigcup_{i=0}^{1} T_{q, i}^{-1}\left(U_{q}\right) \cap J_{q}$ is finite. Therefore by the pigeonhole principle for $m$ sufficiently large, there must exist $i, j \in \mathbb{N}$ with $1 \leq i<j \leq m$ such that

$$
\mathbf{a}^{j}\left(\mathbf{a}^{j-1}\left(\ldots\left(\mathbf{a}^{1}(x)\right) \ldots\right)\right)=\mathbf{a}^{i}\left(\mathbf{a}^{i-1}\left(\ldots\left(\mathbf{a}^{1}(x)\right) \ldots\right)\right) .
$$

Set $\mathbf{b}=\mathbf{a}^{i+1} \cdots \mathbf{a}^{j}, \hat{\mathbf{a}}=\mathbf{a}^{1} \cdots \mathbf{a}^{i}$ and suppose $\hat{\mathbf{a}}(x)=w$, here $w \in \bigcup_{i=0}^{1} T_{q, i}^{-1}\left(U_{q}\right) \cap J_{q}$. Then equation (10) implies that

$$
\mathbf{b}(w)=w
$$

Since $x$ is a $q$ null infinite point so are all its branching points. Thus we have the second property.

To complete our if and only if it suffices to remark that $(i)$ and $(i i)$ imply that $w$ is a $q$ null infinite point. So $q \in \mathcal{B}_{\aleph_{0}}$.

Now we search for all points belonging to $\mathcal{B}_{\aleph_{0}} \cap\left[q_{1}, q_{3}\right]$ by applying Theorem 3.1. Suppose $x$ is a qull infinite point and $x \in J_{q}$. First we point out that

$$
\bigcup_{i=0}^{1} T_{q, i}^{-1}\left(U_{q}\right) \cap J_{q}=\left\{\mathbf{y}_{j}=\Pi\left(01^{j}(10)^{\infty}\right), \mathbf{z}_{j}=\Pi\left(10^{j}(01)^{\infty}\right), 1 \leq j \leq 3\right\}
$$

when $q \in\left[q_{1}, q_{3}\right]$ by Proposition 2.2. By Theorem 3.1, we only need to consider the behavior of elements of $\bigcup_{i=0}^{1} T_{q, i}^{-1}\left(U_{q}\right) \cap J_{q}$ under maps belonging to $\bigcup_{n=0}^{\infty}\left\{T_{q, 0}, T_{q, 1}\right\}^{n}$. Without loss of generality we only need to consider the points $\mathbf{y}_{j}$, for $1 \leq j \leq 3$. We establish the following lemma.

Lemma 3.2. (i) If $q \in\left[q_{1}, q_{3}\right]$ and $\mathbf{y}_{1}$ is a $q$ null infinite point, then

$$
T_{q, 1}\left(\mathbf{y}_{1}\right) \in L_{q}, \quad T_{q, 0}^{j}\left(T_{q, 1}\left(\mathbf{y}_{1}\right)\right) \in L_{q}, j=1,2, \quad T_{q, 0}^{3}\left(T_{q, 1}\left(\mathbf{y}_{1}\right)\right) \in \bigcup_{i=0}^{1} T_{q, i}^{-1}\left(U_{q}\right) \cap J_{q}
$$

(ii) If $q \in\left[q_{1}, q^{\prime}\right)$ then $\mathbf{y}_{2}$ is not a $q$ null infinite point. If $q \in\left[q^{\prime}, q_{3}\right]$ and $\mathbf{y}_{2}$ is a $q$ null infinite point, then

$$
T_{q, 1}\left(\mathbf{y}_{2}\right) \in L_{q}, \quad T_{q, 0}\left(T_{q, 1}\left(\mathbf{y}_{2}\right)\right) \in L_{q}, \quad T_{q, 0}^{2}\left(T_{q, 1}\left(\mathbf{y}_{2}\right)\right) \in \bigcup_{i=0}^{1} T_{q, i}^{-1}\left(U_{q}\right) \cap J_{q} .
$$


Here $q^{\prime}(\approx 1.66184)$ is the positive root of $x^{5}-x^{3}-x^{2}-2 x-2=0$.

(iii)If $q=q_{1}$ and $\mathbf{y}_{3}$ is a $q$ null infinite point, then

$$
T_{q, 1}\left(\mathbf{y}_{3}\right) \in L_{q}, \quad T_{q, 0}\left(T_{q, 1}\left(\mathbf{y}_{3}\right)\right) \in \bigcup_{i=0}^{1} T_{q, i}^{-1}\left(U_{q}\right) \cap J_{q} .
$$

If $q \in\left(q_{1}, q_{3}\right]$ and $\mathbf{y}_{3}$ is a $q$ null infinite point, then $q$ must be $q_{3}$ or $q^{\prime \prime}(\approx 1.67365)$, the positive root of $x^{5}-2 x^{4}+x^{3}-x^{2}+x-1=0$.

Proof. Direct computation yields $(i)$. We prove (ii) now. A simple computation yields

$$
T_{q, 1}\left(\mathbf{y}_{2}\right) \in L_{q}, \quad T_{q, 0}\left(T_{q, 1}\left(\mathbf{y}_{2}\right)\right) \in L_{q}, \quad T_{q, 0}^{2}\left(T_{q, 1}\left(\mathbf{y}_{2}\right)\right) \in\left\{\begin{array}{l}
{\left[\frac{q+q^{2}}{q^{4}-1}, \frac{1+q^{3}}{q^{4}-1}\right] \text { if } q \in\left[q^{\prime}, q_{3}\right]} \\
\left(\frac{1+q^{3}}{q^{4}-1}, \frac{1}{q^{2}-q}\right] \text { if } q \in\left[q_{1}, q^{\prime}\right) .
\end{array}\right.
$$

Here $q^{\prime}(\approx 1.66184)$ is the positive root of $x^{5}-x^{3}-x^{2}-2 x-2=0$. Thus we obtain (12). We now assume $\mathbf{y}_{2}$ is $q$ null infinite for some $q \in\left[q_{1}, q^{\prime}\right)$ and derive a contradiction. If $\mathbf{y}_{2}$ is a $q$ null infinite point for some $q \in\left[q_{1}, q^{\prime}\right)$ then $T_{q, 0}^{2}\left(T_{q, 1}\left(\mathbf{y}_{2}\right)\right) \in \bigcup_{i=0}^{1} T_{q, i}^{-1}\left(U_{q}\right) \cap\left(S_{q} \backslash J_{q}\right)_{R}$ and it can be shown that

$$
T_{q, 0}\left(T_{q, 1}\left(T_{q, 0}^{2}\left(T_{q, 1}\left(\mathbf{y}_{2}\right)\right)\right)\right) \in \bigcup_{i=0}^{1} T_{q, i}^{-1}\left(U_{q}\right) \cap J_{q} .
$$

Therefore by Proposition 2.2 there exists $s \in \bigcup_{i=0}^{1} T_{q, i}^{-1}\left(U_{q}\right) \cap\left(S_{q} \backslash J_{q}\right)_{R}=\left\{\mathbf{y}_{k}, k \geq 4,1 /\left(q^{2}-q\right)\right\}$ and $u \in \bigcup_{i=0}^{1} T_{q, i}^{-1}\left(U_{q}\right) \cap J_{q}$ such that

$$
T_{q, 0}^{2}\left(T_{q, 1}\left(\mathbf{y}_{2}\right)\right)=s \text { and } T_{q, 0}\left(T_{q, 1}\left(T_{q, 0}^{2}\left(T_{q, 1}\left(\mathbf{y}_{2}\right)\right)\right)\right)=u \text {. }
$$

We will show that this is not possible. That is $\mathbf{y}_{2}$ is not a $q$ null infinite point when $q \in\left[q_{1}, q^{\prime}\right)$. In fact,

$$
T_{q, 0}^{2}\left(T_{q, 1}\left(\mathbf{y}_{2}\right)\right)=s
$$

means that

$$
q^{5}-q^{4}-2 q^{3}+2 q+1=q^{-k} .
$$

That is

$$
k=\frac{-\ln \left(q^{5}-q^{4}-2 q^{3}+2 q+1\right)}{\ln q} .
$$

The function $-\ln \left(q^{5}-q^{4}-2 q^{3}+2 q+1\right)(\ln q)^{-1}$ is strictly decreasing on the interval $\left[q_{1}, q^{\prime}\right)$. Table 1 therefore implies that the only possible value of $k$ that may occur within the interval $\left[q_{1}, q^{\prime}\right)$ is $k=4$. It is not possible that $s=1 /\left(q^{2}-q\right)$, since $\lim _{k \rightarrow \infty} \mathbf{y}_{k}=1 /\left(q^{2}-q\right)$ and $-\ln \left(q^{5}-q^{4}-2 q^{3}+2 q+1\right)(\ln q)^{-1}$ is monotonic. Table 1 includes the values of $q$ for which $T_{q, 0}\left(T_{q, 1}\left(T_{q, 0}^{2}\left(T_{q, 1}\left(\mathbf{y}_{2}\right)\right)\right)\right) \in \bigcup_{i=0}^{1} T_{q, i}^{-1}\left(U_{q}\right) \cap J_{q}$. Inspecting Table 1 shows that there are no values of $q$ for which both equations in (14) hold. Therefore we may conclude (ii). 
Table 1: the values of $q$ for $\mathbf{y}_{2}$

\begin{tabular}{lccc}
\hline & $k$ & $q$ & Polynomials \\
\hline$T_{q, 0}^{2}\left(T_{q, 1}\left(\mathbf{y}_{2}\right)\right)=\mathbf{y}_{k}$ & 4 & 1.65027 & $x^{4}-x^{3}-x^{2}-x+1$ \\
& 5 & 1.63923 & $x^{6}-2 x^{4}-2 x^{3}+x+1$ \\
\hline$T_{q, 0}\left(T_{q, 1}\left(T_{q, 0}^{2}\left(T_{q, 1}\left(\mathbf{y}_{2}\right)\right)\right)\right)=\mathbf{y}_{k}$ & 1 & 1.65637 & $x^{5}-2 x^{3}-x^{2}-x-1$ \\
& 2 & 1.64308 & $x^{5}-x^{4}-x^{3}-1$ \\
& 3 & 1.63420 & $x^{7}-2 x^{5}-x^{4}-x^{2}-x-1$ \\
\hline$T_{q, 0}\left(T_{q, 1}\left(T_{q, 0}^{2}\left(T_{q, 1}\left(\mathbf{y}_{2}\right)\right)\right)\right)=\mathbf{z}_{k}$ & 1 & 1.64114 & $x^{2}-x-1$ \\
& 2 & 1.65363 & $x^{5}-2 x^{3}-x^{2}+1$ \\
& 3 & 1.66065 & $x^{5}-x^{4}-x^{3}+1$ \\
\hline
\end{tabular}

It remains to prove $(i i i)$. By direct computation, we have

$$
T_{q, 1}\left(\mathbf{y}_{3}\right) \in L_{q}, \quad T_{q, 0}\left(T_{q, 1}\left(\mathbf{y}_{3}\right)\right) \in\left\{\begin{array}{l}
{\left[\frac{q+q^{2}}{q^{4}-1}, \frac{1+q^{3}}{q^{4}-1}\right] \text { if } q=q_{1}} \\
{\left[\frac{1}{q}, \frac{q+q^{2}}{q^{4}-1}\right) \text { if } q \in\left(q_{1}, q_{3}\right]}
\end{array}\right.
$$

and we obtain (13). Furthermore if $\mathbf{y}_{3}$ is a $q$ null infinite point for some $q \in\left(q_{1}, q_{3}\right]$ then it is straightforward to show that

$$
T_{q, 1}\left(T_{q, 0}\left(T_{q, 0}\left(T_{q, 1}\left(\mathbf{y}_{3}\right)\right)\right)\right) \in \bigcup_{i=0}^{1} T_{q, i}^{-1}\left(U_{q}\right) \cap J_{q} .
$$

Moreover, there must exist $s \in \bigcup_{i=0}^{1} T_{q, i}^{-1}\left(U_{q}\right) \cap\left(S_{q} \backslash J_{q}\right)_{L}=\left\{\mathbf{z}_{k}, k \geq 4,1 / q\right\}$ and $u \in \bigcup_{i=0}^{1} T_{q, i}^{-1}\left(U_{q}\right) \bigcap J_{q}$ such that

$$
T_{q, 0}\left(T_{q, 1}\left(\mathbf{y}_{3}\right)\right)=s \text { and } T_{q, 1}\left(T_{q, 0}\left(T_{q, 0}\left(T_{q, 1}\left(\mathbf{y}_{3}\right)\right)\right)\right)=u .
$$

The equation $T_{q, 0}\left(T_{q, 1}\left(\mathbf{y}_{3}\right)\right)=s$ means that

$$
k=\frac{-\ln \left(1-q^{-1}+q^{2}+q^{3}-q^{4}\right)}{\ln q} .
$$

The function $-\ln \left(1-q^{-1}+q^{2}+q^{3}-q^{4}\right)(\ln q)^{-1}$ is strictly increasing on the interval $\left(q_{1}, q_{3}\right)$ and

$$
\lim _{q \rightarrow q_{3}}-\ln \left(1-q^{-1}+q^{2}+q^{3}-q^{4}\right)(\ln q)^{-1}=+\infty .
$$

Table 2 records the first few solutions of $-\ln \left(1-q^{-1}+q^{2}+q^{3}-q^{4}\right)(\ln q)^{-1}=k$. It is easy to show that the case where $s=1 / q$ is only possible when $q=q_{3}$. In Table 2, we also list the $q$ 's for which $T_{q, 1}\left(T_{q, 0}\left(T_{q, 0}\left(T_{q, 1}\left(\mathbf{y}_{3}\right)\right)\right)\right)=u$ holds. By inspecting Table 2 and using the fact $-\ln \left(1-q^{-1}+q^{2}+q^{3}-\right.$ $\left.q^{4}\right)(\ln q)^{-1}$ is increasing with $q$, we see that the only values of $q$ for which both equations in (15) hold simultaneously are $q \approx 1.67365$ and when $q=q_{3}$.

Table 3 lists the values of $q$ for which equations $(11-13,15)$ hold true independently. In fact, it follows from Lemma 3.2 and the symmetric property of $\mathbf{y}_{k}$ and $\mathbf{z}_{k}$ shown in Lemma 2.1 that equations in (11-13, 15) give all the possible values of $q$ such that $q \in \mathcal{B}_{\aleph_{0}} \cap\left[q_{1}, q_{3}\right]$. 
Table 2: the values of $q$ for $\mathbf{y}_{3}$

\begin{tabular}{lccc}
\hline & $k$ & $q$ & Polynomials \\
\hline$T_{q, 0}\left(T_{q, 1}\left(\mathbf{y}_{3}\right)\right)=\mathbf{z}_{k}$ & 4 & 1.66041 & $x^{6}-x^{5}-x^{3}-x^{2}-1$ \\
& 5 & 1.66883 & $x^{8}-x^{6}-x^{5}-2 x^{4}-x^{3}-x^{2}-x-1$ \\
& 6 & 1.67365 & $x^{5}-2 x^{4}+x^{3}-x^{2}+x-1$ \\
$T_{q, 0}\left(T_{q, 1}\left(\mathbf{y}_{3}\right)\right)=q^{-1}$ & 7 & 1.67644 & $x^{10}-x^{8}-x^{7}-2 x^{6}-x^{5}-x^{4}-x^{3}-x^{2}-x-1$ \\
\hline$T_{q, 1}\left(T_{q, 0}\left(T_{q, 0}\left(T_{q, 1}\left(\mathbf{y}_{3}\right)\right)\right)\right)=\mathbf{y}_{k}$ & 1 & 1.68042 & $x^{5}-x^{4}-x^{3}-x+1$ \\
& 2 & 1.65963 & $x^{5}-x^{4}-x^{3}-x+1$ \\
& 3 & 1.64541 & $x^{7}-2 x^{5}-x^{4}-x^{3}+x+1$ \\
$T_{q, 1}\left(T_{q, 0}\left(T_{q, 0}\left(T_{q, 1}\left(\mathbf{y}_{3}\right)\right)\right)\right)=\mathbf{z}_{k}$ & 1 & 1.65462 & $x^{6}-x^{4}-x^{3}-2 x^{2}-x-1$ \\
& 2 & 1.67365 & $x^{6}-2 x^{4}-x^{3}-1$ \\
& 3 & 1.68400 & $x^{5}-2 x^{4}+x^{3}-x^{2}+x-1$ \\
\hline
\end{tabular}

We are now ready to prove Theorem 1.2.

Proof of Theorem 1.2. By applying Theorem 3.1 and Lemma 3.2, we can find all points belonging to $\mathcal{B}_{\aleph_{0}} \cap\left[q_{1}, q_{3}\right]$ in Table 3 . One can see from Table 3 that

$$
T_{q, 0}\left(T_{q, 1}\left(\mathbf{y}_{3}\right)\right)=\mathbf{z}_{3}, T_{q, 1}\left(T_{q, 0}\left(\mathbf{z}_{3}\right)\right)=\mathbf{y}_{3}, T_{q, 1}\left(T_{q, 0}\left(T_{q, 0}\left(T_{q, 1}\left(\mathbf{y}_{3}\right)\right)\right)\right)=\mathbf{y}_{3}
$$

when $q_{1}(\approx 1.64541)$, which is the positive root of $x^{6}-x^{4}-x^{3}-2 x^{2}-x-1=0$,

$$
T_{q, 0}^{3}\left(T_{q, 1}\left(\mathbf{y}_{1}\right)\right)=\mathbf{z}_{1}, T_{q, 1}^{3}\left(T_{q, 0}\left(\mathbf{z}_{1}\right)\right)=\mathbf{y}_{1}, T_{q, 0}^{3}\left(T_{q, 1}^{4}\left(T_{q, 0}\left(\mathbf{z}_{1}\right)\right)\right)=\mathbf{z}_{1}
$$

when $q_{2}(\approx 1.65462)$, which is the positive root of $x^{6}-2 x^{4}-x^{3}-1=0$, and

$$
T_{q, 0}^{3}\left(T_{q, 1}\left(\mathbf{y}_{1}\right)\right)=\mathbf{y}_{1}
$$

when $q_{3}(\approx 1.68042)$, which is the positive root of $x^{5}-x^{4}-x^{3}-x+1=0$. So, the conditions in Theorem 3.1 are satisfied when $q=q_{1}, q_{2}, q_{3}$, respectively. That is $q_{j} \in \mathcal{B}_{\aleph_{0}}$ for each $1 \leq j \leq 3$.

Finally, it is easy to check that $q \notin \mathcal{B}_{\aleph_{0}}$ if $q$ takes the values listed in Table 3 , except when $q=q_{j}, 1 \leq$ $j \leq 3$. For example, we have

$$
T_{q, 0}^{3}\left(T_{q, 1}\left(\mathbf{y}_{1}\right)\right)=\mathbf{y}_{2}
$$

when $q(\approx 1.65963)$ is the positive root of $x^{5}-x^{4}-x^{3}-x+1=0$. However, there exists no point in $\bigcup_{i=0}^{1} T_{q, i}^{-1}\left(U_{q}\right) \bigcap J_{q}$ such that condition $(i)$ of Theorem 3.1 holds for this value of $q$. The other values of $q$ are dealt with similarly.

\section{Proof of Theorem 1.1}

To prove Theorem 1.1 it suffices to prove 1 has $\aleph_{0} q$-expansions when $q=q_{3}$ and 1 a continuum of $q$-expansions when $q=q_{1}$ and $q=q_{2}$. We only prove the case of $q=q_{1}$, the case of $q=q_{2}$ can be verified in a similar way. 
Table 3: the values of $q$ for $\mathbf{y}_{k}$ and $\mathbf{z}_{k}, 1 \leq k \leq 3$

\begin{tabular}{lccc}
\hline & $k$ & $q$ & Polynomials \\
\hline$T_{q, 0}^{3}\left(T_{q, 1}\left(\mathbf{y}_{1}\right)\right)=\mathbf{y}_{k}\left(\right.$ or $\left.T_{q, 1}^{3}\left(T_{q, 0}\left(\mathbf{z}_{1}\right)\right)=\mathbf{z}_{k}\right)$ & 1 & 1.68042 & $x^{5}-x^{4}-x^{3}-x+1$ \\
& 2 & 1.65963 & $x^{7}-2 x^{5}-x^{4}-x^{3}+x+1$ \\
\hline$T_{q, 0}^{3}\left(T_{q, 1}\left(\mathbf{y}_{1}\right)\right)=\mathbf{z}_{k}\left(\right.$ or $\left.T_{q, 1}^{3}\left(T_{q, 0}\left(\mathbf{z}_{1}\right)\right)=\mathbf{y}_{k}\right)$ & 3 & 1.64541 & $x^{6}-x^{4}-x^{3}-2 x^{2}-x-1$ \\
& 1 & 1.65462 & $x^{6}-2 x^{4}-x^{3}-1$ \\
& 2 & 1.67365 & $x^{5}-2 x^{4}+x^{3}-x^{2}+x-1$ \\
$T_{q, 0}^{2}\left(T_{q, 1}\left(\mathbf{y}_{2}\right)\right)=\mathbf{y}_{k}\left(\right.$ or $\left.T_{q, 1}^{2}\left(T_{q, 0}\left(\mathbf{z}_{2}\right)\right)=\mathbf{z}_{k}\right)$ & 3 & 1.68400 & $x^{8}-2 x^{6}-x^{5}-x^{2}-x-1$ \\
\hline$T_{q, 0}^{2}\left(T_{q, 1}\left(\mathbf{y}_{2}\right)\right)=\mathbf{z}_{k}\left(\right.$ or $\left.T_{q, 1}^{2}\left(T_{q, 0}\left(\mathbf{z}_{2}\right)\right)=\mathbf{y}_{k}\right)$ & 1 & 1.72208 & $x^{4}-x^{3}-x^{2}-x+1$ \\
& 2 & 1.68929 & $x^{6}-2 x^{4}-2 x^{3}+x+1$ \\
& 3 & 1.6663 & $x^{6}-x^{5}-x^{4}-x^{3}+x^{2}+1$ \\
\hline$T_{q, 0}\left(T_{q, 1}\left(\mathbf{y}_{3}\right)\right)=\mathbf{z}_{k}\left(\right.$ or $\left.T_{q, 1}\left(T_{q, 0}\left(\mathbf{z}_{3}\right)\right)=\mathbf{y}_{k}\right)$ & 1 & 1.67602 & $x^{5}-2 x^{3}-x^{2}-1$ \\
\hline$T_{q, 0}\left(T_{q, 1}\left(\mathbf{y}_{3}\right)\right)=\mathbf{z}_{6}, T_{q, 1}\left(T_{q, 0}\left(\mathbf{z}_{6}\right)\right)=\mathbf{z}_{2}$ & 2 & 1.7049 & $x^{5}-x^{4}-x^{3}-1$ \\
$\left(\right.$ or $\left.T_{q, 1}\left(T_{q, 0}\left(\mathbf{z}_{3}\right)\right)=\mathbf{y}_{6}, T_{q, 0}\left(T_{q, 1}\left(\mathbf{y}_{6}\right)\right)=\mathbf{y}_{2}\right)$ & 3 & 1.72004 & $x^{7}-2 x^{5}-x^{4}-x^{2}-x-1$ \\
$T_{q, 0}\left(T_{q, 1}\left(\mathbf{y}_{3}\right)\right)=1 / q, T_{q, 1}\left(T_{q, 0}(1 / q)\right)=\mathbf{y}_{1}$ & 3 & 1.64541 & $x^{6}-x^{4}-x^{3}-2 x^{2}-x-1$ \\
$\left(\right.$ or $\left.T_{q, 1}\left(T_{q, 0}\left(\mathbf{z}_{3}\right)\right)=1 /\left(q^{2}-q\right), T_{q, 0}\left(T_{q, 1}\left(1 /\left(q^{2}-q\right)\right)\right)=\mathbf{z}_{1}\right)$ & & & \\
\hline
\end{tabular}

Theorem 4.1. If $q_{3}(\approx 1.68042)$ is the positive root of $x^{5}-x^{4}-x^{3}-x+1=0$. Then 1 is a $q_{3}$ null infinite point and therefore has $\aleph_{0} q_{3}$-expansions.

Proof. It is straightforward to show that for all $k \geq 0$

$$
\left(T_{q_{3}, 0}^{3} \circ T_{q_{3}, 1}\right)^{k}\left(T_{q_{3}, 1}(1)\right) \in S_{q_{3}} .
$$

Moreover these are the only $\mathbf{a} \in \bigcup_{n=0}^{\infty}\left\{T_{q_{3}, 0}, T_{q_{3}, 1}\right\}^{n}$ such that $\mathbf{a}(1) \in S_{q_{3}}$. This is sufficient to imply that 1 is a $q_{3}$ null infinite point. Namely that for each $k \geq 0$ we have

$$
T_{q_{3}, 0}\left(\left(T_{q_{3}, 0}^{3} \circ T_{q_{3}, 1}\right)^{k}\left(T_{q_{3}, 1}(1)\right)\right)=\Pi\left(1(10)^{\infty}\right) .
$$

Therefore

$$
\Sigma_{q_{3}}(1)=\left\{1\left(10^{3}\right)^{k} 01(10)^{\infty}, 1\left(10^{3}\right)^{\infty}\right\}
$$

Figure 2 demonstrates the construction of $\Sigma_{q_{3}}(1)$.

Theorem 4.2. Let $q_{1}(\approx 1.64541)$ be the positive solution of the equation $x^{6}-x^{4}-x^{3}-2 x^{2}-x-1=0$. Then 1 has $2^{\aleph_{0}} q_{1}$-expansions.

Proof. We proceed via a proof by contradiction. We assume that 1 has $\aleph_{0} q_{1}$-expansions and obtain a contradiction. It is a simple calculation to show that

$$
w:=\left(T_{q_{1}, 0}^{5} \circ T_{q_{1}, 1}^{2}\right)(1) \in S_{q_{1}} .
$$


Figure 2: The construction of $\Sigma_{q_{3}}(1)$

Therefore $w$ has either $\aleph_{0} q_{1}$-expansions or $2^{\aleph_{0}} q_{1}$-expansions. Since we have assumed 1 has $\aleph_{0} q_{1^{-}}$ expansions, $w$ must also have $\aleph_{0} q_{1}$-expansions. Therefore $w$ can be mapped to a $q_{1}$ null infinite point, and by Lemma $C$ it can be mapped to a point with a periodic $q_{1}$-expansion. The above implies that 1 has a $q_{1}$ expansion that begins $\left(1100000\left(\delta_{i}\right)_{i=1}^{\infty}\right)$ where $\left(\delta_{i}\right)_{i=1}^{\infty}$ is eventually periodic. This is obviously equivalent to

$$
1=\frac{1}{q_{1}}+\frac{1}{q_{1}^{2}}+\frac{1}{q_{1}^{7}} \sum_{i=1}^{\infty} \frac{\delta_{i}}{q_{1}^{i}} .
$$

Since $\left(\delta_{i}\right)_{i=1}^{\infty}$ is eventually periodic we may use properties of geometric series to deduce that (17) is equivalent to

$$
1=\frac{1}{q_{1}}+\frac{1}{q_{1}^{2}}+\frac{f\left(q_{1}\right)}{g\left(q_{1}\right)}
$$

where $f(x), g(x) \in \mathbb{Z}[x]$. Equation (18) is just an algebraic relation and so must also be satisfied by the conjugates of $q_{1}$, that is the other roots of $x^{6}-x^{4}-x^{3}-2 x^{2}-x-1=0$. We now show that this cannot be the case for a particular choice of conjugate, namely $q_{1}^{*} \approx-1.20458$. Equation $(18)$ is equivalent to equation (17), so (17) must also hold with $q_{1}$ replaced by $q_{1}^{*}$. We observe the following

$$
\begin{aligned}
1 & =\frac{1}{q_{1}^{*}}+\frac{1}{\left(q_{1}^{*}\right)^{2}}+\frac{1}{\left(q_{1}^{*}\right)^{7}} \sum_{i=1}^{\infty} \frac{\delta_{i}}{\left(q_{1}^{*}\right)^{i}} \\
& \leq \frac{1}{q_{1}^{*}}+\frac{1}{\left(q_{1}^{*}\right)^{2}}+\frac{1}{\left(q_{1}^{*}\right)^{7}} \frac{q_{1}^{*}}{\left(q_{1}^{*}\right)^{2}-1} \\
& <1 .
\end{aligned}
$$

Where the final strict inequality follows from a simple calculation. Thus we have our desired contradiction.

The proof that $q_{2} \notin \mathcal{B}_{1, \aleph_{0}}$ is done analogously. In this case we similarly use a conjugate of $q_{2}$, namely the number $q_{2}^{*} \approx-1.26493$. 


\section{Acknowledgment}

This work was supported by the National Natural Science Foundation of China (NSFC) \#11201312, \#61373087, \#61070087 and \#61272252; the Foundation for Distinguished Young Teachers in Higher Education of Guangdong, China \# Yq2013144; and the Municipal Science and Technology Plan of Shenzhen in China \# JC201105170615A.

\section{References}

[1] S. Baker, On small bases which admit countably many expansions. J. Number Theory 147 (2015), 515-532.

[2] S. Baker, Generalized golden ratios over integer alphabets. Integers 14 (2014), Paper No. A15.

[3] S. Baker and N. Sidorov, Expansions in non-integer bases: lower order revisited. Integers 14 (2014), Paper No. A57.

[4] K. Dajani and M. de Vries, Measures of maximal entropy for random $\beta$-expansions. J. Eur. Math. Soc. 7(2005), 51-68.

[5] K. Dajani and M. de Vries, Invariant densities for random $\beta$-expansions. J. Eur. Math. Soc. 9(2007), 157-176.

[6] K. Dajani and C. Kraaikamp, Random $\beta$-expansions. Ergodic Theory and Dynam. Sys. 23(2003), 461-479.

[7] Z. Daróczy and I. Kátai, Univoque sequences. Publ. Math. Debrecen 42(1993), 397-407.

[8] Z. Daróczy and I. Kátai, On the structure of univoque numbers. Publ. Math. Debrecen 46(1995), 385-408.

[9] M. de Vries and V. Komornik, Unique expansions of real numbers. Adv. Math. 221(2009), 390-427.

[10] P. Erdős, M. Horváth and I. Joó, On the uniqueness of the expansions $1=\sum_{i=1}^{\infty} q^{-n_{i}}$. Acta Math. Hungar. 58(1991), 333-342.

[11] P. Erdős, I. Joó and V. Komornik, Characterization of the unique expansions $1=\sum_{i=1}^{\infty} q^{-n_{i}}$ and related problems. Bull. Soc. Math. Fr. 118(1990), 377-390.

[12] P. Erdös and I. Joó, On the number of expansions $1=\sum q^{-n_{i}}$, II. Ann. Univ. Sci. Budapest. Eötvös Sect. 36(1993), 229-233.

[13] P. Erdös and I. Joó, On the number of expansions $1=\sum q^{-n_{i}}$. Ann. Univ. Sci. Budapest. Eötvös Sect. 35(1992), 129-132. 
[14] P. Erdős and V. Komornik, Developments in non-integer bases. Acta Math. Hungar. 79(1998), 57-83.

[15] P. Glendinning and N. Sidorov, Unique representations of real numbers in non-integer bases. Math. Res. Letters 8(2001), 535-543.

[16] V. Komornik and P. Loreti, Unique developments in non-integer bases. Amer. Math. Monthly 105(1998), 636-639.

[17] W. Parry, On the $\beta$-expansions of real numbers. Acta Math. Acad. Sci. Hung. 11 (1960), 401-416.

[18] N. Sidorov, Almost every number has a continuum of $\beta$-expansions. Amer. Math. Monthly 110(2003), $838-842$.

[19] N. Sidorov, Ergodic-theoretic properties of certain Bernoulli convolutions. Acta Math. Hungar. 101(2003), 345-355.

[20] N. Sidorov, Expansions in non-integer bases: lower, middle and top orders. J. Number Theorem $\mathbf{1 2 9}(2009), 741-754$.

[21] N. Sidorov and A. Vershik, Ergodic properties of the Erdös measure, the entropy of the golden shift, and related problems. Monatsh. Math. 126(1998), 215-261.

[22] Y. R. Zou, J. Lu and W. X. Li, Unique expansion of points of a class of self-similar sets with overlaps. Mathmatika 58(2012), 371-88. 